\title{
DECOMPOSITIONS OF SUBSTOCHASTIC TRANSITION FUNCTIONS
}

\author{
KENNETH LANGE
}

\begin{abstract}
Three decompositions of a substochastic transition function are shown to yield substochastic parts. These are the Lebesgue decomposition with respect to a finite measure, the decomposition into completely atomic and continuous parts, and on $R^{n}$, a decomposition giving a part with continuous distribution function and a part with discontinuous distribution function.
\end{abstract}

Introduction. The present paper considers decomposition theorems for substochastic transition functions. Suppose $\mathscr{A}$ is a $\sigma$-algebra of subsets of a set $Y$. By common usage a substochastic transition function $\mu(y, B)$ means a function from $Y \times \mathscr{A}$ into the unit interval satisfying: (i) for fixed $B \in \mathscr{A}, y \rightarrow \mu(y, B)$ is $\mathscr{A}$-measurable, (ii) for fixed $y \in Y, \mu(y, \cdot)$ is a measure on $\mathscr{A}$.

Now for each $y$ there are several possible decompositions of $\mu(y, \cdot)$. If $v$ is a finite measure on $\mathscr{A}$, then $\mu(y, \cdot)$ has a Lebesgue decomposition with respect to $v$. If $\mathscr{A}$ is countably generated, it makes sense to speak of splitting $\mu(y, \cdot)$ into completely atomic and continuous parts. Finally, if the underlying space is Euclidean and $\mathscr{A}$ is the collection of Borel sets, it is possible to decompose $\mu(y, \cdot)$ uniquely into a part having continuous distribution function and a part having discontinuous distribution function, as spelled out in Corollary 4 . The point of this paper is to prove that each such decomposition yields substochastic pieces. The combination, in Corollary 9, of these separate decompositions gives a more or less complete breakdown of a substochastic transition function on Euclidean space into four substochastic pieces.

Preliminaries and definitions. We wish to modify slightly the definition of substochastic transition function so as to achieve somewhat greater generality and to avoid pathological cases which we are unable to handle.

Received by the editors March 3, 1972 and, in revised form, April 20, 1972.

AMS (MOS) subject classifications (1970). Primary 60G05, 60J35; Secondary 28A20.

Key words and phrases. Substochastic transition function, space of measures, Lebesgue decomposition, atom, distribution function, Fell topology.

(c) American Mathematical Society 1973 
To do this let us introduce some terminology for Borel spaces and spaces of measures.

Borel spaces. A set $X$ together with a distinguished $\sigma$-algebra of subsets $\mathscr{A}$ will be called a Borel space. The sets in $\mathscr{A}$ will be called Borel sets. Often $X$ will be termed the Borel space and $\mathscr{A}$ tacitly understood. For instance, if $X$ is a topological space, $\mathscr{A}$ will always be taken to be the smallest $\sigma$-algebra containing the open sets. $(X, \mathscr{A})$ is said to be countably generated if $\mathscr{A}$ is the smallest $\sigma$-algebra containing some sequence of Borel sets $B_{1}, B_{2}, \cdots$. A function $f: X \rightarrow Y$ between two Borel spaces $(X, \mathscr{A})$ and $(Y, \mathscr{B})$ is called Borel if $f^{-1}(B) \in \mathscr{A}$ whenever $B \in \mathscr{B}$. Note that composition of Borel maps yields a Borel map.

Spaces of measures. Suppose $X$ is a countably generated Borel space. $M_{1}(X)$ will denote the collection of measures $\mu$ on the Borel sets of $X$ satisfying the requirement $\mu(X) \leqq 1$. As in [3] give to $M_{1}(X)$ the smallest Borel structure making each of the maps $\mu \rightarrow \mu(B)$ Borel for $B$ a Borel set of $X$. Then $M_{1}(X)$ is a countably generated Borel space.

If the underlying space $X$ is Polish (complete, separable, metric) an alternative description of the Borel sets of $M_{1}(X)$ can be given. Let $C(X)$ be the bounded, continuous, real-valued functions on $X$ equipped with the sup norm topology. Then $M_{1}(X)$ can be viewed as a subset of the dual of $C(X)$ equipped with the weak* topology. In other words, $M_{1}(X)$ has the weakest topology making each of the maps $\mu \rightarrow \int f d \mu$ continuous for $f \in C(X)$. It is known (perhaps not well known) that $M_{1}(X)$ is a Polish space under the weak* topology. (Imitate the treatment of spaces of probability measures given in Chapter 2 of [6]. Keep in mind that a net $\mu_{\alpha} \rightarrow \mu$ weak* iff $\lim \sup _{\alpha} \mu_{\alpha}(C) \leqq \mu(C)$ for each closed set $C$ and $\lim _{\alpha} \mu_{\alpha}(X)=\mu(X)$ iff $\liminf _{\alpha} \mu_{\alpha}(V) \leqq \mu(V)$ for each open set $V$ and $\lim _{\alpha} \mu_{\alpha}(X)=\mu(X)$.) According to Lemma 2.3 of [7] the Borel structure associated with the weak* topology coincides with the Borel structure for $M_{1}(X)$ introduced above.

Substochastic transition function. Let $X$ be a countably generated Borel space. A Borel map $y \rightarrow \mu(y, \cdot)$ from a Borel space $Y$ into $M_{1}(X)$ will be termed a substochastic transition function.

Fell topology. Suppose $X$ is a locally compact Polish space. It will be convenient to define a topology on the collection of closed subsets $\mathscr{C}(X)$ of $X$. This topology is determined by the basis of open sets $U(C: \mathscr{F})=$ $\{Z \in \mathscr{C}(X): Z \cap C=\varnothing, Z \cap V \neq \varnothing$ if $V \in \mathscr{F}\}$, where $C$ is compact in $X$ and $\mathscr{F}$ is a finite (possibly empty) collection of open sets. $\mathscr{C}(X)$ is a compact metric space with this topology. See [4] and $\S 4$ of [5] for more details. A map $f: T \rightarrow \mathscr{C}(X)$ from a topological space $T$ into $\mathscr{C}(X)$ will be called upper semicontinuous if $\{t \in T: f(t) \cap C=\varnothing\}$ is open in $T$ for each compact set $C$ of $X$. 
Decomposition results. Our first theorem was originally established by Doob in a slightly different form using a martingale convergence argument. See Example 2.7 of the supplement of [2].

THEOREM 1. Suppose $X$ is a countably generated Borel space and $y \rightarrow$ $\mu(y, \cdot) \in M_{1}(X)$ and $y \rightarrow v(y, \cdot) \in M_{1}(X)$ are substochastic transition functions. For each y let $\mu(y, \cdot)=\mu_{s}(y, \cdot)+\mu_{a b}(y, \cdot)$ be the Lebesgue decomposition of $\mu(y, \cdot)$ with respect to $\nu(y, \cdot)$, i.e. $\mu_{s}(y, \cdot) \perp v(y, \cdot)$ and $\mu_{a b}(y, \cdot) \ll$ $\nu(y, \cdot)$. Then $y \rightarrow \mu_{s}(y, \cdot)$ and $y \rightarrow \mu_{a b}(y, \cdot)$ are substochastic.

Proof. The map $y \rightarrow(\mu(y, \cdot), v(y, \cdot))$ is Borel into $M_{1}(X) \times M_{1}(X)$. Since the map $M_{1}(X) \times M_{1}(X) \rightarrow M_{1}(X)$ taking a pair of measures $(\omega, \eta)$ into the singular part of $\omega$ with respect to $\eta$ is Borel (Theorem 2.10 of [3]), $y \rightarrow \mu_{s}(y, \cdot)$ is a composition of Borel maps, and hence Borel. That $y \rightarrow \mu_{a b}(y, \cdot)$ is Borel follows by subtraction.

THEOREM 2. Suppose $X$ is a countably generated Borel space and $y \rightarrow \mu(y, \cdot) \in M_{1}(X)$ is a substochastic transition function. For each $y$ decompose $\mu(y, \cdot)=\mu_{a}(y, \cdot)+\mu_{c}(y, \cdot)$ into a completely atomic measure $\mu_{a}(y, \cdot)$ and an atom free measure $\mu_{c}(y, \cdot)$. Then $y \rightarrow \mu_{a}(y, \cdot)$ and $y \rightarrow \mu_{c}(y, \cdot)$ are substochastic.

Proof. Mimic the proof of the last theorem and apply Theorem 2.12 of [3].

We now turn to transition functions on Euclidean $n$-space $R^{n}$. Recall that the distribution function $F_{\mu}$ of finite measure $\mu$ on the Borel sets of $R^{n}$ is defined as $F_{\mu}(z)=\mu\left(\left(-\infty, z_{1}\right] \times \cdots \times\left(-\infty, z_{n}\right]\right)$, where $z=$ $\left(z_{1}, \cdots, z_{n}\right) \in R^{n}$. For $1 \leqq i \leqq n$ let $\pi_{i}: R^{n} \rightarrow R$ be the projection of a point in $R^{n}$ onto its $i$ th coordinate. Given a constant $c, \pi_{i}^{-1}(c)$ will be a hyperplane perpendicular to one of the axes.

THEOREM 3. The distribution function $F_{\mu}$ of a measure $\mu \in M_{1}\left(R^{n}\right)$ is continuous iff no hyperplane of the form $\pi_{i}^{-1}(c)$ has positive $\mu$-mass.

Proof. The condition is clearly necessary. For sufficiency fix $x \in R^{n}$ and write

where

$$
F_{\mu}(x)-F_{\mu}(y)=\sum_{i=1}^{n} F_{\mu}\left(z^{i}\right)-F_{\mu}\left(z^{i-1}\right)
$$

$$
\begin{aligned}
z_{l}^{i}=x_{l} & \text { if } i \geqq l, \\
=y_{l} & \text { if } i<l .
\end{aligned}
$$

If $\|x-y\| \leqq \delta$, then $\left|F_{\mu}\left(z^{i}\right)-F_{\mu}\left(z^{i-1}\right)\right| \leqq \mu\left(\pi_{i}^{-1}\left(\left[x_{i}-\delta, x_{i}+\delta\right]\right)\right)$. For $\varepsilon>0$, $\delta>0$ can be selected so that $\mu\left(\pi_{i}^{-1}\left(\left[x_{i}-\delta, x_{i}+\delta\right]\right)\right) \leqq \varepsilon / n$ for each $i$. 
COROllaRY 4. Every measure $\mu \in M_{1}\left(R^{n}\right)$ has a decomposition $\mu=$ $\mu_{1}+\mu_{2}$, where $\mu_{1}$ has continuous distribution function and $\mu_{2}$ is either the zero measure or has discontinuous distribution function. This decomposition is unique in the sense that if $\mu=\mu_{1}^{\prime}+\mu_{2}^{\prime}$ is a second such decomposition, then either $\mu_{1}=\mu_{1}^{\prime}$ or $\mu_{1}\left(R^{n}\right)>\mu_{1}^{\prime}\left(R^{n}\right)$.

Proof. Let $W$ be the finite or countable union of all hyperplanes of the form $\pi_{i}^{-1}(c)$ with positive $\mu$-mass. Put $\mu_{1}(B)=\mu(B \backslash W)$ and $\mu_{2}(B)=$ $\mu(B \cap W)$ for each Borel set $B$.

Definition. For each $\varepsilon>0$ let $d_{\varepsilon}: M_{1}\left(R^{n}\right) \rightarrow \mathscr{C}\left(R^{n}\right)$ be the map taking a measure $\mu$ into the union of the hyperplanes of the form $\pi_{i}^{-1}(c)$ having $\mu$-mass $\geqq \varepsilon$.

Lemma 5. $d_{\varepsilon}: M_{1}\left(R^{n}\right) \rightarrow \mathscr{C}\left(R^{n}\right)$ is a Borel map.

Proof. According to Lemma 4.2 of [5] it suffices to show $d_{\varepsilon}$ upper semicontinuous. Let $K$ be a compact set of $R^{n}$ and consider $\left\{\mu: d_{\varepsilon}(\mu) \cap\right.$ $K \neq \varnothing\}$. Let us show that this set is closed in $M_{1}\left(R^{n}\right)$. Suppose $\mu_{m} \rightarrow \mu$ and $d_{\varepsilon}\left(\mu_{m}\right) \cap K \neq \varnothing$. There is at least one $i$, say $i=1$, and a subsequence $\left\{\mu_{m_{l}}\right\}$ from $\left\{\mu_{m}\right\}$ such that $\pi_{1}^{-1}\left(c_{m_{l}}\right) \cap K \neq \varnothing$ and $\mu_{m_{l}}\left(\pi_{1}^{-1}\left(c_{m_{l}}\right)\right) \geqq \varepsilon$ for all $l$ and some choice of constants $c_{m_{l}}$. Take $x_{m_{l}} \in \pi_{1}^{-1}\left(c_{m_{l}}\right) \cap K$. By passing to a subsequence if necessary, we can assume $x_{m_{l}} \rightarrow x \in K$. To finish the proof it is enough to prove $\mu\left(\pi_{1}^{-1}\left(\pi_{1}(x)\right)\right) \geqq \varepsilon$. But this follows because for each $\delta>0$,

$$
\begin{aligned}
\mu\left(\pi _ { 1 } ^ { - 1 } \left(\left[\pi_{1}(x)-\right.\right.\right. & \left.\left.\left.\delta, \pi_{1}(x)+\delta\right]\right)\right) \\
& \geqq \lim \sup \mu_{m_{l}}\left(\pi_{1}^{-1}\left(\left[\pi_{1}(x)-\delta, \pi_{1}(x)+\delta\right]\right)\right) \geqq \varepsilon .
\end{aligned}
$$

Lemma 6. Suppose $X$ is a locally compact Polish space. The map $(\mu, A) \rightarrow \mu(A)$ is Borel from $M_{1}(X) \times \mathscr{C}(X) \rightarrow[0,1]$. (Compare 3.9 or [3].)

Proof. Choose a metric $d$ on $X$ giving the topology and satisfying $0 \leqq d \leqq 1$. For $A$ closed in $X$ put $d(y, A)=\inf _{x \in A} d(y, x)$. Define for each positive integer $m$ a map from $X \times \mathscr{C}(X) \rightarrow[0,1]$ by taking $(y, A) \rightarrow$ $(1-d(y, A))^{m}$. This map is clearly continuous in $y$ for fixed $A$. Furthermore, it is Borel in $A$ for fixed $y$. Indeed, $A \rightarrow d(y, A)$ is a Borel map because, for each real $t>0,\{A \in \mathscr{C}(X): d(y, A)<t\}=\{A \in \mathscr{C}(X):\{z: d(y, z)<t\} \cap$ $A \neq \varnothing\}$. It now follows that $(y, A) \rightarrow(1-d(y, A))^{m}$ is jointly Borel.

Next consider the map $(\mu, A) \rightarrow \int(1-d(y, A))^{m} d \mu(y)$. Since $y \rightarrow d(y, A)$ is a continuous, bounded function, $(\mu, A) \rightarrow \int(1-d(y, A))^{m} d \mu(y)$ is continuous in $\mu$ for fixed $A$. But by Fubini's theorem and the above argument $(\mu, A) \rightarrow \int(1-d(y, A))^{m} d \mu(y)$ is Borel in $A$ for fixed $\mu$. Hence the map is jointly Borel. Since $\mu(A)=\inf _{m} \int(1-d(y, A))^{m} d \mu(y)$, the statement of the lemma is rendered obvious. 
Lemma 7. Suppose $X$ is a topological space with countably generated Borel structure. Let $y \rightarrow \mu(y, \cdot)$ be a map from a Borel space $Y$ into $M_{1}(X)$. Then a necessary and sufficient condition for $y \rightarrow \mu(y, \cdot)$ to be substochastic is that $y \rightarrow \mu(y, A)$ be Borel for each closed (resp. open) set $A$.

Proof. Clearly it is enough to treat only the case of closed sets. Necessity is immediate. For sufficiency let $\mathscr{D}$ be the collection of Borel sets $B$ for which $y \rightarrow \mu(y, B)$ is Borel. $\mathscr{D}$ contains $X$ and every closed set. If $B_{1}, B_{2} \in \mathscr{D}$ and $B_{1} \supset B_{2}$, then $B_{1} \mid B_{2} \in \mathscr{D}$. If $\left\{B_{m}\right\}_{m=1}^{\infty}$ is an increasing sequence of sets from $\mathscr{D}$, then $\bigcup_{m=1}^{\infty} B_{m} \in \mathscr{D}$. Hence $\mathscr{D}$ is a Dynkin system in the terminology of [1]. Since the collection of closed sets is closed under finite intersections, $\mathscr{D}$ contains the smallest $\sigma$-algebra generated by the closed sets, and thus coincides with the Borel sets. (See Theorem 4.12 of [1].)

THEOREM 8. Suppose $y \rightarrow \mu(y, \cdot) \in M_{1}\left(R^{n}\right)$ is a substochastic transition function. Then the decomposition described in Corollary 4 for each $y$ is substochastic.

Proof. It suffices to show that the map $\mu \rightarrow \mu_{2}$ from $M_{1}\left(R^{n}\right)$ into $M_{1}\left(R^{n}\right)$ is Borel. According to Lemma 7 we need merely prove $\mu \rightarrow \mu_{2}(A)$ Borel for $A$ closed. Now $\mu_{2}(A)=\sup _{k} \mu\left(d_{1 / k i}(\mu) \cap A\right)$ indicates that it is enough to prove $\mu \rightarrow \mu\left(d_{1 / k}(\mu) \cap A\right)$ is Borel. But $\mu \rightarrow\left(\mu, d_{1 / k}(\mu), A\right) \rightarrow(\mu$, $\left.d_{1 / k}(\mu) \cap A\right) \rightarrow \mu\left(d_{1 / k}(\mu) \cap A\right)$ is a composition of Borel maps due to Lemmas 5 and 6 and the fact that the intersection map $\mathscr{C}\left(R^{n}\right) \times \mathscr{C}\left(R^{n}\right) \rightarrow$ $\mathscr{C}\left(R^{n}\right)$ is Borel. (See Lemma 4.4 of [5].)

COROllaRy 9. Suppose $y \rightarrow \mu(y, \cdot) \in M_{1}\left(R^{n}\right)$ is a substochastic transition function. Then $\mu(y, \cdot)$ has a decomposition into four substochastic parts; $\mu(y, \cdot)=\mu_{a}(y, \cdot)+\mu_{s 1}(y, \cdot)+\mu_{s 2}(y, \cdot)+\mu_{a b}(y, \cdot)$, where for each $y:$

(i) $\mu_{a}(y, \cdot)$ is completely atomic.

(ii) $\mu_{s 1}(y, \cdot)$ is nonatomic, singular with respect to Lebesgue measure, and has continuous distribution function.

(iii) $\mu_{s 2}(y, \cdot)$ is nonatomic, singular with respect to Lebesgue measure, and either has discontinuous distribution function or is the zero measure.

(iv) $\mu_{a b}(y, \cdot)$ is absolutely continuous with respect to Lebesgue measure. Furthermore, this decomposition is unique in the obvious sense.

\section{BIBLIOGRAPHY}

1. R. B. Ash, Measure, integration, and functional analysis, Academic Press, New York, 1972.

2. J. L. Doob, Stochastic processes, Wiley, New York; Chapman \& Hall, London, 1953. MR 15, 445. 
3. L. Dubins and D. Freedman, Measurable sets of measures, Pacific J. Math. 14 (1964), 1211-1222. MR 30 \#4887.

4. J. M. G. Fell, $A$ Hausdorff topology for the closed subsets of a locally compact nonHausdorff space, Proc. Amer. Math. Soc. 13 (1962), 472-476. MR 25 \#2573.

5. K. Lange, Borel sets of probability measures, Pacific J. Math. (to appear).

6. K. R. Parthasarathy, Probability measures on metric spaces, Probability and Math. Statist., no. 3, Academic Press, New York, 1967. MR 37 \#2271.

7. V. S. Varadarajan, Groups of automorphisms of Borel spaces, Trans. Amer. Math. Soc. 109 (1963), 191-220. MR 28 \#3139.

Department of Mathematics, University of New Hampshire, Durham, New HAMPSHIRE 03824

Current address: Department of Biomathematics, University of California, Los Angeles, California 90024 